\title{
INTEGRATION OF RS AND GIS FOR STUDYING THE GEOMORPHOLOGICAL AND PEDOLOGICAL FEATURES OF SOME SOILS SOUTH WEST OF EL-SADAT CITY, EGYPT
}

\author{
M.S. Amira(1), M.H. Bahnassy(2), W.M. $\mathrm{Nada}^{(1)}$, F.E. Abu $\mathrm{Agwa}^{(1)}$ \\ and Doaa M. Ahmed(1) \\ (1) Soil Sci. Dept., Fac. Agric. Menoufia univ. \\ (2) Soil \& Water Dept., Fac. Agric., El-Shatby, Alexandria Univ.
}

Received: Oct. 26, 2019

Accepted: Oct. 29,2019

\begin{abstract}
The current work aims to study the geomorphological and pedological characteristics as well as classification for soils in the area located at south west of ElSadat City. The integration of Remote Sensing (RS) and Geographic Information System (GIS) techniques was used to perform this work. This work could be presented important information about the potentiality of these soils for proper plans of reclamation, improvement and management.
\end{abstract}

The geomorphic map produced using RS and GIS technology indicated that, there are three identified and interpreted geomorphic units in this area. These units are Low Terraces, Moderate Terraces and High Terraces.

Fourteen soil profiles were selected representing these geomorphic units. The land and site features are observed and registered. Profiles were morphologically described, and then samples were collected representing the subsequent layers in each profile for integrated physical and chemical analyses.

The elevation of the studied profiles varied between 14 and $52 \mathrm{~m}$. that increased from the Low Terraces to the High ones. The soils have almost flat to gently undulating topography with gentle sloping. All studied soils are deep and characterized as freely well drained.

These soils have almost slightly to gravelly loamy sand texture with rapid hydraulic conductivity. The soils have mainly weak granular to subangular structure and some layers have single grains. The most of studied soils are virgin without or with scanty vegetation.

The morphological rating scale (relative distinctness of horizons "RHD" and relative profile development "RPD") indicates a slight distinctness between horizons which mainly attributed to the depositional pattern and lor regimes of soil materials more than development.

The analytical data of the studied soils revealed that, the soil reaction is slightly alkaline. All the studied soils are non-saline and not sodic. The soils differ from slightly to strongly calcareous according their $\mathrm{CaCO}_{3}$ content. Organic matter (OM) and gypsum were low. The cation exchange capacity (CEC) was also low due to the low content of fine fractions and $O M$ in these soils.

The studied soils haven't any diagnostic horizons, therefore they were classified up to sub great group level under Entisols order mainly as Typic Torripsaments.

Key words: RS, GIS, geomorphic units, pedological features, soil classification.

INTRODUCTION

The rapid growth of the Egyptian population on the limited natural resources in the Nile Valley and Delta and the increase in per capita requirements have caused a sharp decline in the self- 
sufficient rate of major food commodities (Ashoub, 1996). Besides population pressure, industrialization, construction and cultural development consumed large areas of agricultural land. Therefore, an urgent demand for horizontal expansion in the Egyptian deserts is indispensable besides the vertical expansion of presently cultivated area. The Egyptian government has lunched several ambitious land development projects. The major objectives of these projects are to increase the inhabited area from $7 \%$ to $25 \%$ of the total area of Egypt over the next 10 years. This could be achieved through the reclamation and development of about $2.4-2.9$ million feddan in the fringes of the Nile valley and Delta as well as in the desert regions (Abdel-Ghaffar et al., 1997 and Quinn, 1998)

Fringes west of the Nile Delta are considered very important for these projects. These fringes have the most of best potentially suitable agricultural land resources for future expansion and development in Egypt. One of the susceptible lands occurs mainly in the fringes of the Nile valley and Delta, such as the area around El Sadat City.

According to Erian (1989) and Soil Survey Staff (2014), the soil temperature regime in this part from the north of Western Desert could be considered as "Thermic". The soil moisture regime is mainly "Aridic" or Torric.

Remote sensing (RS) is now recognized as an important tool in monitoring and managing natural resources (Lillesand and Kiefer, 2007). They added that RS technique is one of the important methods that used for soil survey, mapping and environmental investigation.

ESRI (2003) stated that, geographic information system (GIS) is a system for the management, analysis, and display of geographic information, which is represented by a series of geographic datasets that model geography using simple, generic data structures.

Integration of RS and GIS play a major role in both soil survey and soil mapping applications. The development of methods to map soil properties using optical RS data in combination with field measurements has been the objective of several studies during the last decade (Dehaan and Taylor, 2003).

The aim of the present work is to furnish a comprehensive study on geomorphological and pedological features including classification of the soils in the area south west of El-Sadat City using the integration of remote sensing (RS) and geographic information system (GIS) techniques. This work could be presented important information served for promising plans of reclamation, improvement and management of these soils.

\section{MATERIALS AND METHODS \\ Location of the study area}

The studied area is located at south west of El-Sadat City. It is located between latitudes $30^{\circ} 15^{\prime} 50^{\prime \prime}$ to $30^{\circ} 34^{\prime} 00^{\prime \prime} \mathrm{N}$; and longitudes $30^{\circ} 46^{\prime} 1.396 "$ to $30^{\circ} 54^{\prime} 44.017^{\prime \prime} \mathrm{E}$ (Fig., 1). The studied area occupied an area of $53.47 \mathrm{Km}^{2}$ (13216.62 feddan).

The studied area is characterized by semi-arid to arid climatic condition (desert condition) with dry and hot summer season and warm winter with low precipitation of about $10 \mathrm{~mm} / \mathrm{year}$ (Erian, 1989).

\section{Geomorphology and soil mapping using RS and GIS \\ Geomorphologic map was carried out} using digital image processing of Land Sat 8.0 $\mathrm{ETM}^{+}$image dated 2017, executed using ENVI software 5.0 (ITT, 2012). Image was stretched using linear $2 \%$, smoothly filtered, and their histograms were 
matched according to Lillesand and Kiefer (2007). Image was atmospherically corrected using FLAASH module (ITT, 2012). GIS works were performed to produce geomorphologic map for the studied area using Arc GIS software 10.1 (ESRI, 2014).

\section{Field work}

Fourteen soil profiles were chosen representing the different geomorphic units of the studied area. Longitudes, latitudes and elevation were defined in the field using GPS apparatus. The land and site features are observed, described and registered according to FAO (2006). Soil profiles were dug deep down to about 120 cm. and morphologically described according to FAO (2006). Soil color was determined in dry and moist samples using the Munsell Color (1992). The important morphological features such as soil color, texture, structure, consistence and the boundary between horizons were used for evaluating the pedological development according to Bilzi and Ciolkosz (1977). Soil samples were collected from each horizon of the soil profiles and were air dried. Particles with a diameter less than $2 \mathrm{~mm}$ were used for the physio-chemical analyses.

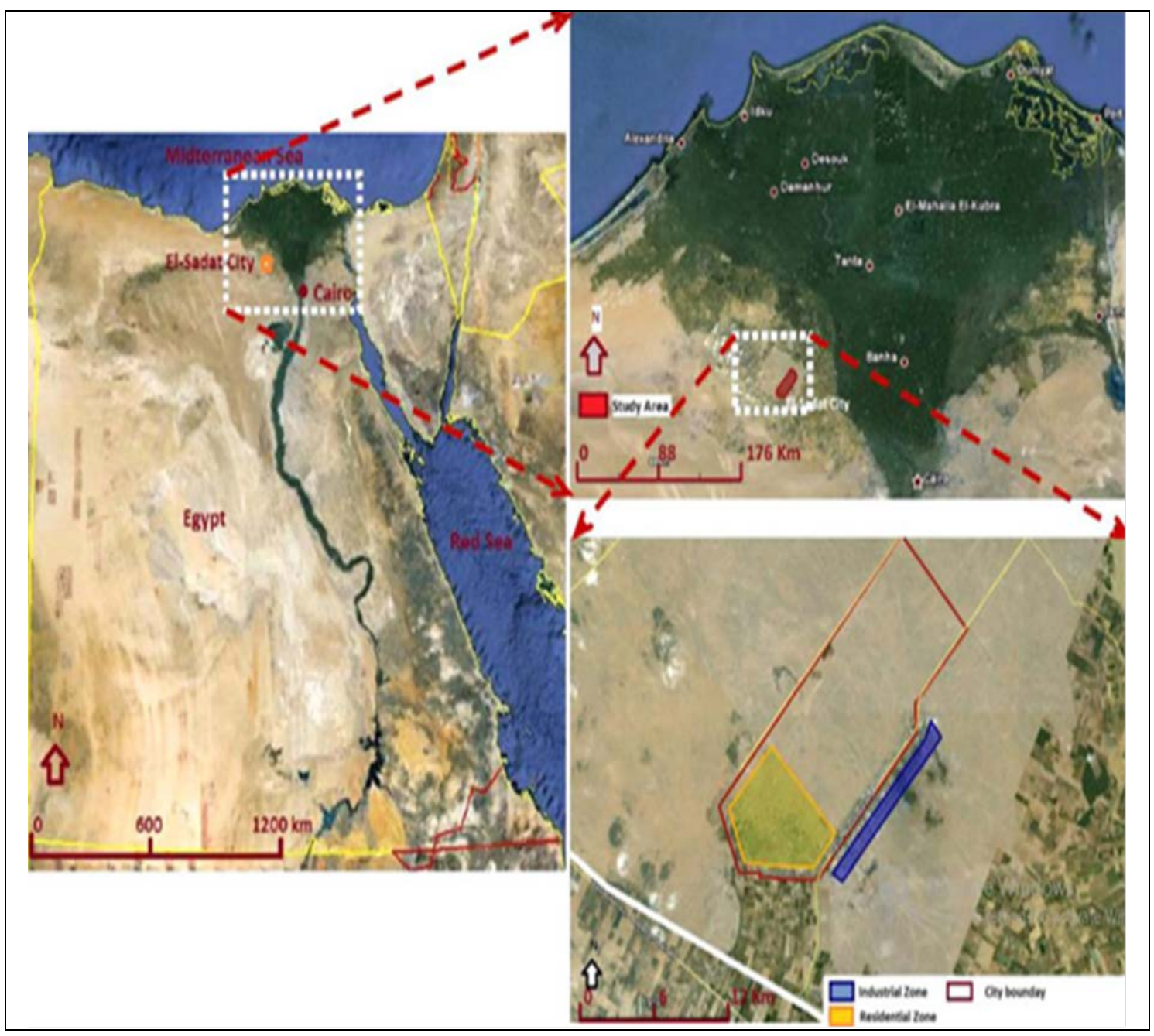

Fig. (1): Location of the study area at south west of El- Sadat City. 


\section{Laboratory analyses}

Gravels content, particle size distribution, electrical conductivity (EC), $\mathrm{pH}$, organic matter (OM), calcium carbonate $\left(\mathrm{CaCO}_{3}\right)$, gypsum, cation exchange capacity (CEC) and exchangeable $\mathrm{Na}^{+}$percentage (ESP), were determined according to Burt and Soil Survey Staff (2014). The soil hydraulic conductivity was determined at saturation under a constant head (Klute and Dirksen, 1986).

\section{Soil classification}

The soils of the studied area were classified down to sub great group level according to Soil Survey Staff (2014).

\section{RESULTUS AND DISCUSSION Geomorphology}

Satellite image interpretation indicated that, the investigated area could be considered as a Pediplain sediments including three main geomorphic units namely: 1) low Terraces, 2) Moderate Terraces and high Terraces. These unites and location of their representative soil profiles are presented in Fig. (2). Area of each unit in $\mathrm{km}^{2}$, feddan, and \% from the total studied area are presented in Table (1).

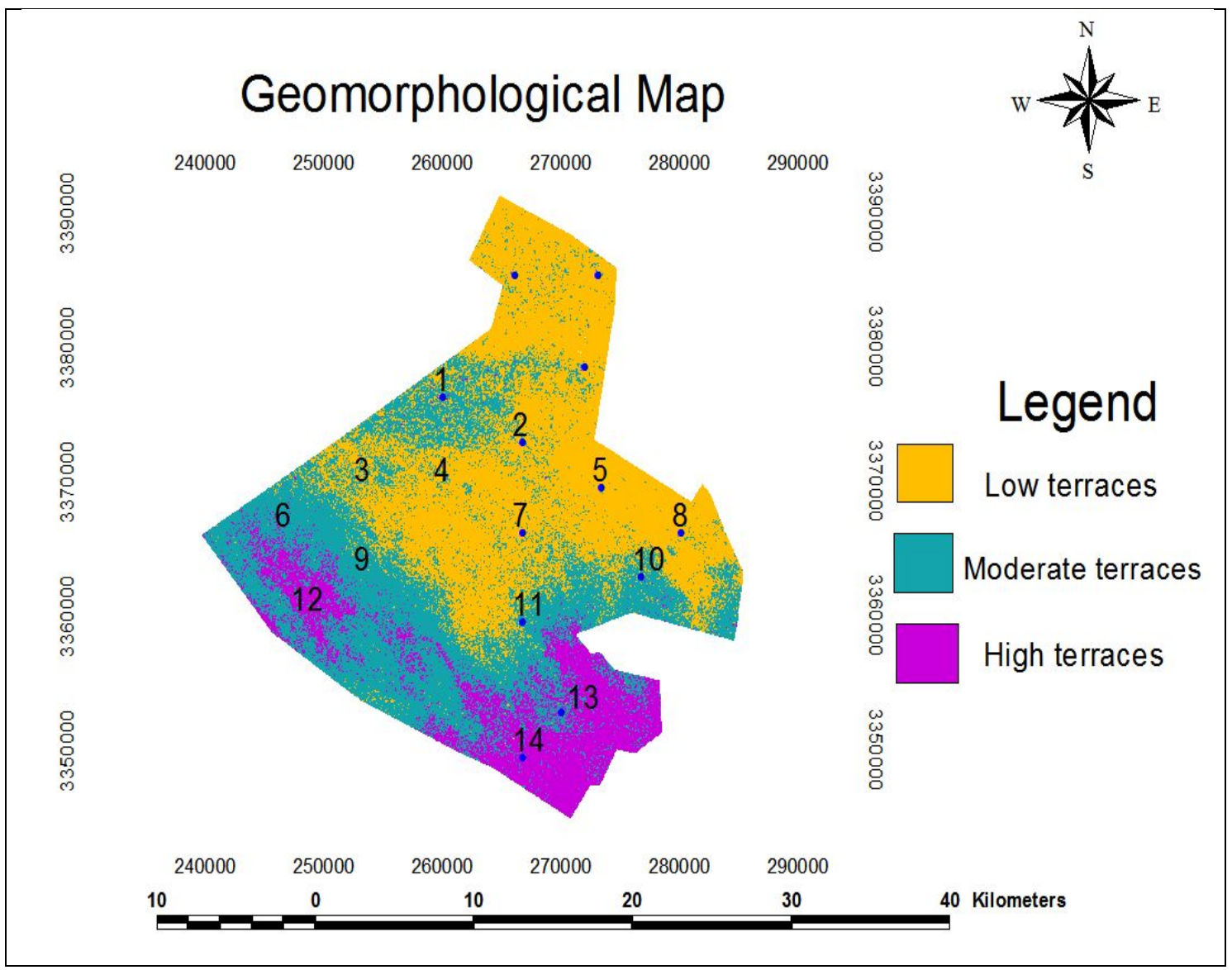

Fig. (2): Geomorphic unites and location of studied profiles. 
Integration of RS and GIS for studying the geomorphological and ....................

Table (1): Area of each geomorphic unit and its \% from the total study area.

\begin{tabular}{|c|c|c|c|}
\hline \multirow{2}{*}{ Geomorphic units } & \multicolumn{3}{|c|}{ Area } \\
\cline { 2 - 4 } & $\mathrm{Km}^{2}$ & Feddan & $\%$ \\
\hline Low Terraces & 23.23 & 5741.58 & 43.44 \\
\hline Moderate Terraces & 17.36 & 4290.55 & 32.46 \\
\hline High Terraces & 12.88 & 3184.49 & 24.1 \\
\hline Total & 53.47 & 13216.62 & 100 \\
\hline
\end{tabular}

\section{Soil morphology and rating scale}

The morphological features of the studied soils (Table, 2 ) revealed that, the elevation of the studied profiles showed increasing values from the Low Terraces (profiles 2, 4, 5, 7 and 8) to the High ones (profiles 12, 13 1nd 14). It varies between 14 and $30 \mathrm{~m}$. a.s.l. for the Low Terraces; 34 to $39 \mathrm{~m}$. a.s.l. for the Moderate; and 42 to $52 \mathrm{~m}$. a.s.l. for the High ones. The soils have almost flat to gently undulating with gentle sloping topography. Land surface and all layers of the studied soils have different contents of gravels increased in the direction from Low to High Terraces. All studied soils are deep and characterized as freely well drained. The main hue notation of studied soil color is around brown degrees (10YR to 7.5YR). These soils have almost slightly to gravelly sandy loam texture throughout their depths with mainly weak granular to subangular blocky structure. Some layers have single grains sand. They are slightly to highly calcareous having soft to hard (dry) and friable (moist) consistency and the sandy layers are loose. The most of studied soils are virgin without or with scanty vegetation.

Findings of Bilzi and Ciolkosz (1977) for the morphological rating scale can be used to compare adjacent horizons to give a comparison of the relative distinctness of horizons (RHD). Also, it can be used to compare horizons in the solum to the $C$ horizon in order to give a relative profile development (RPD) evaluation. The morphological rating scale (RHD and RPD) presented in Table (3) showed a relatively low to moderate values indicating a slight distinctness between horizons and weak profile development. The relative differences between values between layers are mostly corresponded with color and could be mainly attributed to the stratification and depositional pattern of soil materials more than development.

\section{Physiochemical properties}

The analytical data of studied soils (Table 4) show that, these soils have mainly slightly gravelly to gravelly sandy loam texture as indicating from the whole profiles means (wpm). They are nonsaline as indicated by their EC values that they are lower than $4 \mathrm{dSm}^{-1}$ (as a wpm). Soil reaction is slightly alkaline as indicated by $\mathrm{pH}$ values where they are < 8.0 in all profile's layers. Total carbonate $\left(\mathrm{CaCO}_{3}\right)$ content indicated that these soils varied between slightly calcareous in the Low Terraces (that have 3 to $7.7 \%$ as wpm) and moderately to strongly calcareous in the Moderate and High Terraces (that have mostly $>10 \%$ as w.p.m.). Gypsum content is very low $(<1 \%)$. Also, organic matter (OM) is very low owing to the prevailing aridity of the region and its scanty vegetation. The cation exchange capacity (CEC) is low dependent on the fine fractions and organic matter contents. The ESP values are lower than $5 \%$ indicating non sodicity effect in all studied soils. 
M.S. Amira, et al.,

Table (2): Morphological features of the studied soil profiles.

\begin{tabular}{|c|c|c|c|c|c|c|c|c|c|}
\hline \multirow{2}{*}{$\begin{array}{c}\text { Geomorphic } \\
\text { units }\end{array}$} & \multirow{2}{*}{$\begin{array}{l}\text { Profile } \\
\text { No. }\end{array}$} & \multirow{2}{*}{$\begin{array}{c}\text { Elevat-ion } \\
\text { m asl }\end{array}$} & \multirow{2}{*}{$\begin{array}{l}\text { Depth } \\
\text { cm }\end{array}$} & \multicolumn{2}{|c|}{ Color } & \multirow{2}{*}{ Structure $^{1}$} & \multicolumn{2}{|c|}{ Consistence $^{2}$} & \multirow{2}{*}{ Boundary ${ }^{3}$} \\
\hline & & & & Dry & Moist & & Dry & Moist & \\
\hline \multirow{5}{*}{ 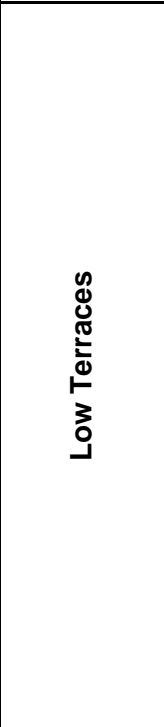 } & 2 & +29 & $\begin{array}{c}0-30 \\
30-60 \\
60-90 \\
90-120\end{array}$ & $\begin{array}{l}\text { 10YR 7/4 } \\
\text { 10YR 7/3 } \\
\text { 10YR 7/4 } \\
\text { 10YR 7/4 }\end{array}$ & $\begin{array}{l}6 / 3 \\
6 / 3 \\
6 / 3 \\
6 / 3\end{array}$ & $\begin{array}{c}\text { 1f sbk } \\
\text { 1f sbk } \\
\text { sg } \\
\text { sg }\end{array}$ & $\begin{array}{c}\text { soft } \\
\text { soft } \\
\text { loose } \\
\text { loose }\end{array}$ & $\begin{array}{c}v \text { friable } \\
\text { v friable } \\
\text { loose } \\
\text { loose }\end{array}$ & $\begin{array}{c}\text { diffuse } \\
\text { diffuse } \\
\text { diffuse } \\
-\end{array}$ \\
\hline & 4 & +27 & $\begin{array}{c}0-30 \\
30-60 \\
60-90 \\
90-120\end{array}$ & $\begin{array}{l}\text { 10YR 7/4 } \\
\text { 10YR 7/4 } \\
\text { 10YR 7/4 } \\
\text { 10YR 7/4 }\end{array}$ & $\begin{array}{l}6 / 4 \\
6 / 4 \\
6 / 4 \\
6 / 4\end{array}$ & $\begin{array}{l}\text { sg } \\
\text { sg } \\
\text { sg } \\
\text { sg }\end{array}$ & $\begin{array}{l}\text { loose } \\
\text { loose } \\
\text { loose } \\
\text { loose }\end{array}$ & $\begin{array}{l}\text { loose } \\
\text { loose } \\
\text { loose } \\
\text { loose }\end{array}$ & $\begin{array}{c}\text { diffuse } \\
\text { diffuse } \\
\text { diffuse } \\
-\end{array}$ \\
\hline & 5 & +18 & \begin{tabular}{|c|}
$0-20$ \\
$20-50$ \\
$50-80$ \\
$80-110$ \\
$110-140$ \\
\end{tabular} & $\begin{array}{l}\text { 10YR 6/4 } \\
\text { 10YR 6/6 } \\
\text { 10YR 6/6 } \\
\text { 10YR 7/4 } \\
\text { 10YR 7/4 }\end{array}$ & $\begin{array}{l}5 / 4 \\
5 / 6 \\
5 / 6 \\
6 / 4 \\
6 / 4\end{array}$ & $\begin{array}{c}2 \mathrm{~m}, \mathrm{f} \text { sbk to gr } \\
2 \mathrm{c}, \mathrm{m} \text { gbk } \\
1 \mathrm{~m}, \mathrm{f} \text { sbk to } \mathrm{gr} \\
1 \mathrm{f} \mathrm{sbk} \text { to } \mathrm{gr} \\
\mathrm{sg}\end{array}$ & $\begin{array}{c}\text { soft } \\
\text { soft } \\
\text { soft } \\
\text { soft } \\
\text { loose }\end{array}$ & $\begin{array}{c}\text { friable } \\
\text { v friable } \\
\text { v friable } \\
\text { loose } \\
\text { loose }\end{array}$ & $\begin{array}{c}\text { gradual s } \\
\text { diffuse } \\
\text { gradual s } \\
\text { diffuse } \\
-\end{array}$ \\
\hline & 7 & +30 & \begin{tabular}{|c|}
$0-30$ \\
$30-60$ \\
$60-90$ \\
$90-120$
\end{tabular} & $\begin{array}{l}\text { 10YR 6/6 } \\
\text { 10YR 6/6 } \\
\text { 10YR 6/4 } \\
\text { 10YR 7/4 }\end{array}$ & $\begin{array}{l}6 / 4 \\
5 / 6 \\
5 / 4 \\
6 / 3\end{array}$ & $\begin{array}{c}1 \mathrm{f} \text { sbk to } g r \\
2 \mathrm{~m}, \mathrm{f} \text { sbk to } \mathrm{gr} \\
1 \mathrm{f} \text { sbk to } g r \\
\mathrm{sg}\end{array}$ & $\begin{array}{c}\text { soft } \\
\text { soft } \\
\text { soft } \\
\text { loose }\end{array}$ & $\begin{array}{c}v \text { friable } \\
v \text { friable } \\
v \text { friable } \\
\text { loose }\end{array}$ & $\begin{array}{c}\text { Diffuse } \\
\text { Diffuse } \\
\text { gradual s } \\
-\end{array}$ \\
\hline & 8 & +14 & \begin{tabular}{|c|}
$0-20$ \\
$20-50$ \\
$50-100$ \\
$100-130$ \\
\end{tabular} & $\begin{array}{l}7.5 Y R 6 / 4 \\
7.5 Y R 6 / 6 \\
7.5 Y R \text { 6/4 } \\
7.5 Y R 6 / 4\end{array}$ & $\begin{array}{l}5 / 4 \\
5 / 6 \\
5 / 4 \\
5 / 4\end{array}$ & $\begin{array}{c}1 \mathrm{~m}, \mathrm{f} g r \\
1 \mathrm{~m}, \mathrm{f} \text { sbk to } \mathrm{gr} \\
2 \mathrm{~m}, \mathrm{f} \text { sbk to } \mathrm{gr} \\
1 \mathrm{~m}, \mathrm{f} \text { sbk to } \mathrm{gr}\end{array}$ & $\begin{array}{c}\text { soft } \\
\text { hard } \\
\text { hard } \\
\text { soft }\end{array}$ & $\begin{array}{c}\text { friable } \\
\text { friable } \\
\text { friable } \\
\mathrm{v} \text { friable }\end{array}$ & $\begin{array}{c}\text { gradual s } \\
\text { Clear } \\
\text { Diffuse } \\
-\end{array}$ \\
\hline \multirow{6}{*}{ 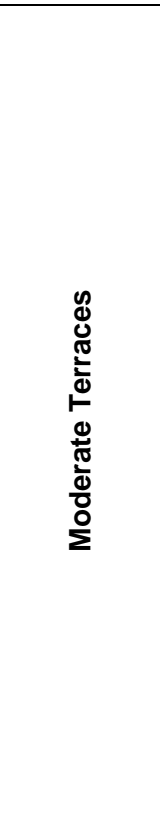 } & 1 & +35 & \begin{tabular}{c|}
$0-30$ \\
$30-60$ \\
$60-90$ \\
$90-120$
\end{tabular} & $\begin{array}{l}\text { 10YR 6/6 } \\
\text { 10YR 6/6 } \\
\text { 10YR 7/4 } \\
\text { 10YR 7/4 }\end{array}$ & $\begin{array}{l}5 / 6 \\
5 / 6 \\
6 / 4 \\
6 / 4\end{array}$ & $\begin{array}{l}1 \mathrm{~m}, \mathrm{f} \text { sbk to } \mathrm{gr} \\
1 \mathrm{~m}, \mathrm{f} \text { sbk to } \mathrm{gr} \\
1 \mathrm{~m}, \mathrm{f} \text { sbk to } \mathrm{gr} \\
1 \mathrm{~m}, \mathrm{f} \text { sbk to } \mathrm{gr}\end{array}$ & $\begin{array}{l}\text { hard } \\
\text { hard } \\
\text { hard } \\
\text { loose }\end{array}$ & $\begin{array}{c}\text { friable } \\
\text { friable } \\
\text { friable } \\
\text { loose }\end{array}$ & $\begin{array}{c}\text { Diffuse } \\
\text { gradual s } \\
\text { Diffuse } \\
-\end{array}$ \\
\hline & 3 & +38 & $\begin{array}{c}0-30 \\
30-60 \\
60-90 \\
90-120 \\
\end{array}$ & $\begin{array}{l}\text { 10YR 6/6 } \\
\text { 10YR 6/6 } \\
\text { 10YR 6/6 } \\
\text { 10YR 7/6 }\end{array}$ & $\begin{array}{l}5 / 4 \\
5 / 6 \\
5 / 6 \\
6 / 6\end{array}$ & $\begin{array}{c}1 \mathrm{~m}, \mathrm{f} \text { sbk to } \mathrm{gr} \\
1 \mathrm{~m}, \mathrm{f} \mathrm{sbk} \text { to } \mathrm{gr} \\
1 \mathrm{~m}, \mathrm{f} \text { sbk to } \mathrm{gr} \\
\mathrm{sg}\end{array}$ & $\begin{array}{c}\text { soft } \\
\text { soft } \\
\text { loose } \\
\text { loose }\end{array}$ & $\begin{array}{c}\text { friable } \\
\text { friable } \\
\text { loose } \\
\text { loose }\end{array}$ & $\begin{array}{c}\text { diffuse } \\
\text { diffuse } \\
\text { gradual s } \\
-\end{array}$ \\
\hline & 6 & +39 & $\begin{array}{c}0-30 \\
30-60 \\
60-90 \\
90-120 \\
\end{array}$ & $\begin{array}{l}7.5 Y R 6 / 4 \\
7.5 Y R \text { 6/4 } \\
7.5 Y R \text { 7/6 } \\
7.5 Y R \text { 6/4 }\end{array}$ & $\begin{array}{l}5 / 4 \\
5 / 4 \\
6 / 4 \\
5 / 4\end{array}$ & $\begin{array}{c}1 \mathrm{f} \mathrm{sbk} \text { to } \mathrm{gr} \\
1 \mathrm{~m}, \mathrm{f} \mathrm{sbk} \text { to } \mathrm{gr} \\
\mathrm{sg} \\
1 \mathrm{~m}, \mathrm{f} \mathrm{gr}\end{array}$ & $\begin{array}{c}\text { soft } \\
\text { soft } \\
\text { loose } \\
\text { soft }\end{array}$ & $\begin{array}{c}\text { friable } \\
\mathrm{v} \text { friable } \\
\text { loose } \\
\mathrm{v} \text { friable }\end{array}$ & $\begin{array}{c}\text { Diffuse } \\
\text { gradual s } \\
\text { gradual s } \\
-\end{array}$ \\
\hline & 9 & +39 & $\begin{array}{c}0-30 \\
30-60 \\
60-90 \\
90-120\end{array}$ & $\begin{array}{l}7.5 Y R \text { 7/6 } \\
7.5 Y R \text { 6/6 } \\
7.5 Y R \text { 6/6 } \\
7.5 Y R \text { 6/6 }\end{array}$ & $\begin{array}{l}5 / 4 \\
5 / 4 \\
5 / 6 \\
5 / 6\end{array}$ & $\begin{array}{c}1 \mathrm{~m}, \mathrm{f} \text { sbk to } \mathrm{gr} \\
1 \mathrm{~m}, \mathrm{f} \mathrm{gr} \\
1 \mathrm{~m}, \mathrm{f} \text { sbk to } \mathrm{gr} \\
1 \mathrm{~m}, \mathrm{f} \text { sbk to } \mathrm{gr}\end{array}$ & $\begin{array}{l}\text { hard } \\
\text { hard } \\
\text { hard } \\
\text { soft }\end{array}$ & $\begin{array}{c}\text { friable } \\
\text { friable } \\
\text { friable } \\
v \text { friable }\end{array}$ & $\begin{array}{c}\text { gradual s } \\
\text { Diffuse } \\
\text { Diffuse } \\
-\end{array}$ \\
\hline & 10 & +34 & $\begin{array}{c}0-20 \\
20-50 \\
50-80 \\
80-120\end{array}$ & $\begin{array}{l}\text { 10YR 6/4 } \\
\text { 10YR 6/6 } \\
\text { 10YR 6/6 } \\
\text { 10YR 7/6 }\end{array}$ & $\begin{array}{l}5 / 4 \\
5 / 6 \\
5 / 6 \\
6 / 4\end{array}$ & $\begin{array}{l}2 \mathrm{~m}, \mathrm{f} \text { sbk to } g r \\
2 \mathrm{~m}, \mathrm{f} \text { sbk to } g r \\
2 \mathrm{~m}, \mathrm{f} \text { sbk to } g r \\
1 \mathrm{f} \text { sbk to } g r\end{array}$ & $\begin{array}{c}\text { soft } \\
\text { hard } \\
\text { hard } \\
\text { soft }\end{array}$ & $\begin{array}{c}\text { friable } \\
\text { firm } \\
\text { friable } \\
\mathrm{v} \text { friable }\end{array}$ & $\begin{array}{c}\text { gradual s } \\
\text { Diffuse } \\
\text { gradual s } \\
-\end{array}$ \\
\hline & 11 & +39 & $\begin{array}{c}0-30 \\
30-60 \\
60-90 \\
90-120\end{array}$ & $\begin{array}{l}7.5 Y R 6 / 6 \\
7.5 Y R \text { 6/6 } \\
7.5 Y R 6 / 6 \\
7.5 Y R 6 / 6\end{array}$ & $\begin{array}{l}5 / 6 \\
5 / 6 \\
5 / 6 \\
5 / 6\end{array}$ & $\begin{array}{l}1 \mathrm{~m}, \mathrm{f} \text { sbk to } \mathrm{gr} \\
1 \mathrm{~m}, \mathrm{f} \text { sbk to } \mathrm{gr} \\
1 \mathrm{~m}, \mathrm{f} \text { sbk to } \mathrm{gr} \\
1 \mathrm{~m}, \mathrm{f} \text { sbk to } \mathrm{gr}\end{array}$ & $\begin{array}{l}\text { soft } \\
\text { soft } \\
\text { soft } \\
\text { loose }\end{array}$ & $\begin{array}{c}\text { friable } \\
\text { friable } \\
\text { friable } \\
\text { loose }\end{array}$ & $\begin{array}{c}\text { diffuse } \\
\text { diffuse } \\
\text { diffuse } \\
-\end{array}$ \\
\hline \multirow{3}{*}{ 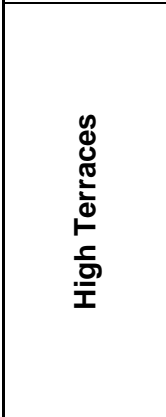 } & 12 & +42 & $\begin{array}{c}0-30 \\
30-60 \\
60-90 \\
90-120\end{array}$ & $\begin{array}{l}7.5 Y R \text { 6/6 } \\
7.5 Y R \text { 6/6 } \\
7.5 Y R \text { 6/4 } \\
7.5 Y R \text { 6/4 }\end{array}$ & $\begin{array}{l}5 / 6 \\
5 / 6 \\
5 / 4 \\
5 / 4\end{array}$ & $\begin{array}{c}2 \mathrm{~m}, \mathrm{f} \text { sbk to } g r \\
2 \mathrm{~m}, \mathrm{f} \text { sbk to } g r \\
2 \mathrm{~m}, \mathrm{f} \text { sbk to } \mathrm{gr} \\
2 \mathrm{~m}, \mathrm{f} \text { sbk }\end{array}$ & $\begin{array}{l}\text { soft } \\
\text { soft } \\
\text { hard } \\
\text { hard }\end{array}$ & $\begin{array}{l}\text { friable } \\
\text { friable } \\
\text { friable } \\
\text { friable }\end{array}$ & $\begin{array}{c}\text { Diffuse } \\
\text { gradual s } \\
\text { Diffuse } \\
-\end{array}$ \\
\hline & 13 & +46 & $\begin{array}{c}0-30 \\
30-60 \\
60-90 \\
90-120\end{array}$ & $\begin{array}{l}\text { 10YR 6/6 } \\
\text { 10YR 6/6 } \\
\text { 10YR 6/6 } \\
\text { 10YR 6/6 }\end{array}$ & $\begin{array}{l}5 / 6 \\
5 / 6 \\
5 / 6 \\
5 / 6\end{array}$ & $\begin{array}{c}2 \mathrm{~m}, \mathrm{f} \text { sbk } \\
1 \mathrm{~m}, \mathrm{f} \mathrm{sbk} \text { to } \mathrm{gr} \\
1 \mathrm{~m}, \mathrm{f} \text { sbk to } \mathrm{gr} \\
1 \mathrm{~m}, \mathrm{f} \text { sbk to } \mathrm{gr}\end{array}$ & $\begin{array}{l}\text { soft } \\
\text { soft } \\
\text { soft } \\
\text { soft }\end{array}$ & $\begin{array}{c}\text { Friable } \\
\text { v friable } \\
\text { v friable } \\
\text { v friable }\end{array}$ & $\begin{array}{l}\text { diffuse } \\
\text { diffuse } \\
\text { diffuse } \\
-\end{array}$ \\
\hline & 14 & +52 & $\begin{array}{c}0-30 \\
30-60 \\
60-90 \\
90-120 \\
\end{array}$ & $\begin{array}{l}7.5 Y R \text { 6/6 } \\
7.5 Y R \text { 6/6 } \\
7.5 Y R 6 / 6 \\
7.5 Y R \text { 6/6 }\end{array}$ & $\begin{array}{l}5 / 6 \\
5 / 6 \\
5 / 6 \\
5 / 6\end{array}$ & $\begin{array}{l}1, \mathrm{f} \text { sbk to } \mathrm{gr} \\
1 \mathrm{~m}, \mathrm{f} \mathrm{sbk} \text { to } \mathrm{gr} \\
1 \mathrm{f} \text { sbk to } \mathrm{gr} \\
1 \mathrm{f} \mathrm{sbk} \text { to } \mathrm{gr}\end{array}$ & $\begin{array}{l}\text { Hard } \\
\text { soft } \\
\text { soft } \\
\text { soft }\end{array}$ & $\begin{array}{c}\text { friable } \\
\text { friable } \\
\text { friable } \\
\mathrm{v} \text { friable }\end{array}$ & $\begin{array}{l}\text { diffuse } \\
\text { diffuse } \\
\text { diffuse } \\
-\end{array}$ \\
\hline
\end{tabular}

Abbreviations: Texture*: L=loamy, $\mathrm{S}=$ sandy, $\mathrm{s}$ g=slightly gravely, g=gravely; Structure ${ }^{1}$ : 1=weak, 2 =moderate, $v=$ very, $\mathrm{f}=$ fine, $\mathrm{m}=$ medium, $\mathrm{co}=$ coarse, $\mathrm{gr}=$ granular, $\mathrm{sbk}=$ subangular blocky; Consistence ${ }^{2}$ :

$\mathrm{s}=$ slightly, $v=$ very, $\mathrm{x}=$ extremely; Boundary ${ }^{3}: \mathrm{s}=$ smooth 
Table (3): Morphological rating scale (RHD and RPD) for studied soil profiles.

\begin{tabular}{|c|c|c|c|c|c|c|c|c|c|c|c|c|c|c|c|c|c|c|c|}
\hline \multirow{2}{*}{$\stackrel{\frac{n}{2}}{5}$} & \multirow{2}{*}{$\begin{array}{l}P . \\
N^{\circ}\end{array}$} & \multirow{2}{*}{ Transition } & \multirow{2}{*}{ 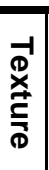 } & \multirow{2}{*}{ 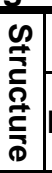 } & \multicolumn{2}{|c|}{ Color } & \multicolumn{2}{|c|}{ Consistence } & \multirow{2}{*}{ 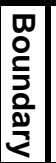 } & \multirow{2}{*}{ 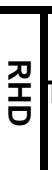 } & \multirow{2}{*}{ Transition } & & \multirow{2}{*}{ 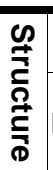 } & \multicolumn{2}{|c|}{ Color } & \multicolumn{2}{|c|}{ Consistence } & \multirow{2}{*}{ 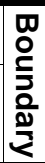 } & \multirow{2}{*}{ गु } \\
\hline & & & & & & Moist & Dry & Moist & & & & & & Dry & Moist & Dry & Moist & & \\
\hline \multirow{16}{*}{ 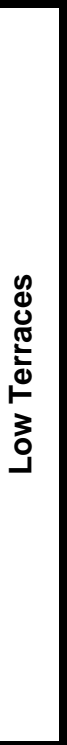 } & & $1^{\text {st/ } / 2^{\text {nd }}}$ & 0 & 0 & 1 & 0 & 0 & 0 & 0 & 1 & $1^{\text {st/Last }}$ & 0 & 3 & 0 & 0 & 1 & 1 & 0 & 5 \\
\hline & 2 & $2^{\text {nd }} / 3^{\text {rd }}$ & 0 & 3 & 1 & 0 & 1 & 1 & 0 & 6 & $2^{\text {nd }} /$ Last & 0 & 3 & 1 & 0 & 1 & 1 & 0 & 6 \\
\hline & & $3^{\text {rd }} / 4^{\text {th }}$ & 0 & 0 & 0 & 0 & 0 & 0 & 0 & 0 & $3^{\text {rd }} /$ Last & 0 & 0 & 0 & 0 & 0 & 0 & 0 & 0 \\
\hline & & $1^{\mathrm{st} / 2^{\text {nd }}}$ & 0 & 0 & 0 & 0 & 0 & 0 & 0 & 0 & $1^{\text {st }} /$ Last & 0 & 1 & 0 & 0 & 0 & 0 & 0 & 1 \\
\hline & 4 & $2^{\text {nd }} / 3^{\text {rd }}$ & 0 & 0 & 0 & 0 & 0 & 0 & 0 & 0 & $2^{\text {nd }} /$ Last & 0 & 1 & 0 & 0 & 0 & 0 & 0 & 1 \\
\hline & & $3^{\text {rd }} / 4^{\text {th }}$ & 0 & 1 & 0 & 0 & 0 & 0 & 0 & 1 & $3^{\text {rd } / \text { Last }}$ & 0 & 1 & 0 & 0 & 0 & 0 & 0 & 1 \\
\hline & & $1^{\mathrm{st}} / 2^{\text {nd }}$ & 0 & 1 & 2 & 2 & 0 & 1 & 1 & 7 & $1^{\text {st }} /$ Last & 0 & 3 & 1 & 1 & 1 & 2 & 1 & 9 \\
\hline & & $2^{\text {nd }} / 3^{\text {rd }}$ & 0 & 2 & 0 & 0 & 0 & 1 & 1 & 4 & $2^{\text {nd }} /$ Last & 0 & 3 & 3 & 3 & 1 & 1 & 0 & 11 \\
\hline & 5 & $3^{\text {rd }} / 4^{\text {th }}$ & 0 & 1 & 3 & 3 & 0 & 0 & 1 & 8 & $3^{\text {rd }}$ /Last & 0 & 3 & 3 & 3 & 1 & 1 & 1 & 12 \\
\hline & & $4^{\text {rd }} / 5^{\text {th }}$ & 0 & 3 & 0 & 0 & 1 & 1 & 0 & 5 & $4^{\text {rd } / \text { Last }}$ & 0 & 3 & 0 & 0 & 1 & 1 & 0 & 5 \\
\hline & & $1^{\mathrm{st} / 2^{\text {nd }}}$ & 0 & 2 & 0 & 3 & 0 & 0 & 0 & 5 & $1^{\text {st }} /$ Last & 0 & 3 & 3 & 1 & 1 & 1 & 0 & 9 \\
\hline & 7 & $2^{\text {nd }} / 3^{\text {rd }}$ & 0 & 2 & 2 & 2 & 0 & 0 & 1 & 7 & $2^{\text {nd }} /$ Last & 0 & 3 & 3 & 4 & 1 & 1 & 0 & 12 \\
\hline & & $3^{\text {rd }} / 4^{\text {th }}$ & 0 & 3 & 1 & 2 & 1 & 1 & 1 & 9 & $3^{\text {rd }} /$ Last & 0 & 3 & 1 & 2 & 1 & 1 & 1 & 9 \\
\hline & & $1^{\mathrm{st} / 2^{\text {nd }}}$ & 1 & 1 & 2 & 2 & 2 & 0 & 1 & 9 & $1^{\text {st }} /$ Last & 1 & 1 & 0 & 0 & 0 & 1 & 1 & 4 \\
\hline & 8 & $2^{\text {nd }} / 3^{\text {rd }}$ & 0 & 1 & 2 & 2 & 0 & $\mathbf{0}$ & 2 & 7 & $2^{\text {nd }} /$ Last & 0 & 0 & 2 & 2 & 2 & 1 & 2 & 9 \\
\hline & & $3^{\text {rd }} / 4^{\text {th }}$ & 0 & 1 & 0 & 0 & 2 & 1 & 0 & 4 & $3^{\text {rd }}$ /Last & 0 & 1 & 0 & 0 & 2 & 1 & 0 & 4 \\
\hline & & $1^{\text {st } / 2^{\text {nd }}}$ & 0 & 0 & 0 & $\overline{0}$ & $\overline{0}$ & $\overline{0}$ & 1 & 1 & $1^{\text {st }} /$ Last & 0 & 0 & 3 & 3 & 3 & 2 & 0 & 11 \\
\hline & 1 & $2^{\text {nd } / 3^{\text {rd }}}$ & 0 & 0 & 3 & 3 & 0 & 0 & 1 & 7 & $2^{\text {nd }} /$ Last & 0 & 0 & 3 & 3 & 3 & 2 & 1 & 12 \\
\hline & & $3^{\text {rd }} / 4^{\text {th }}$ & 0 & 0 & 0 & 0 & 3 & 2 & 0 & 5 & $3^{\text {rd }} /$ Last & 0 & 0 & 0 & 0 & 3 & 2 & 0 & 5 \\
\hline & & $1^{\text {st } / 2^{\text {nd }}}$ & 1 & 0 & $\overline{0}$ & 2 & $\overline{0}$ & $\overline{0}$ & 0 & 3 & $1^{\text {st }}$ Last & 0 & 3 & 1 & 3 & 1 & 2 & 0 & 10 \\
\hline & 3 & $2^{\text {nd }} / 3^{\text {rd }}$ & 1 & 1 & 0 & 0 & 1 & 2 & 1 & 6 & $2^{\text {nd } / \text { Last }}$ & 1 & 3 & 1 & 1 & 1 & 2 & 0 & 9 \\
\hline & & $3^{\text {rd }} / 4^{\text {th }}$ & 0 & 3 & 1 & 1 & 0 & 0 & 1 & 6 & $3^{\text {rd } / \text { Last }}$ & 0 & 3 & 1 & 1 & 0 & 0 & 1 & 6 \\
\hline$\stackrel{0}{0}$ & & $1^{\text {st } / 2^{\text {nd }}}$ & 0 & 1 & 0 & 0 & 0 & & 1 & 3 & $1^{\text {st }} /$ Last & 0 & 2 & 0 & $\overline{0}$ & 0 & 1 & 0 & 3 \\
\hline & 6 & $2^{\text {nd } / 3^{\text {rd }}}$ & 0 & 3 & 1 & 1 & & & 0 & 7 & st & 0 & 1 & 0 & & & & 1 & 2 \\
\hline & & $3^{\text {rd }} / 4^{\text {th }}$ & 0 & 3 & 1 & 1 & 1 & 1 & 1 & 8 & $3^{\text {rd/Last }}$ & 0 & 3 & 1 & 1 & 1 & 1 & 1 & 8 \\
\hline$\stackrel{ \pm}{\pi}$ & & $1^{\text {st } / 2^{\text {nd }}}$ & 0 & 1 & 3 & 0 & 0 & & 1 & 5 & $1^{\text {st/Last }}$ & 0 & 0 & 3 & 2 & & 1 & 1 & 9 \\
\hline$\check{\vdots}$ & 9 & $2^{\text {nd }} / 3^{\text {rd }}$ & 0 & 1 & 0 & 2 & 0 & 0 & 0 & 3 & $2^{\text {nd/Last }}$ & 0 & 1 & 0 & 2 & 2 & 1 & 0 & 6 \\
\hline & & $3^{\text {rd }} / 4^{\text {th }}$ & 0 & 0 & 0 & 0 & 2 & 1 & 0 & 3 & $3^{\text {rd }}$ /Last & 0 & 0 & 0 & 0 & 2 & 1 & 0 & 3 \\
\hline & & $1^{\text {st }} / 2^{\text {nd }}$ & 1 & 0 & 0 & 0 & 0 & 0 & 0 & 1 & $1^{\text {st/Last }}$ & 0 & 1 & 0 & 0 & 1 & 2 & 0 & 4 \\
\hline & 10 & $2^{\text {nd }} / 3^{\text {rd }}$ & 1 & 0 & 0 & 0 & 0 & 0 & 0 & 1 & $2^{\text {nd } / \text { Last }}$ & 1 & 1 & 0 & 0 & 1 & 2 & 0 & 5 \\
\hline & & $3^{\text {rd }} / 4^{\text {th }}$ & 0 & 1 & 0 & 0 & 1 & 2 & 0 & 4 & $3^{\text {rd }} /$ Last & 0 & 1 & 0 & 0 & 1 & 2 & 0 & 4 \\
\hline & & $1^{\text {st } / 2^{\text {nd }}}$ & 0 & 0 & $\overline{2}$ & $\overline{2}$ & $\overline{2}$ & & 1 & 8 & $1^{\text {st } / \text { Last }}$ & 1 & 2 & 3 & 1 & $\overline{0}$ & 1 & 1 & 9 \\
\hline & 11 & $2^{\text {nd }} / 3^{\text {rd }}$ & 0 & 0 & 0 & 0 & 0 & & 1 & 2 & $2^{\text {nd }} /$ Last & 1 & 2 & 1 & 3 & 2 & & 0 & 11 \\
\hline & & $3^{\text {rd }} / 4^{\text {th }}$ & 1 & 2 & 1 & 3 & 2 & 1 & 1 & 11 & $3^{\text {rd } / \text { Last }}$ & 1 & 2 & 1 & 3 & 2 & 1 & 1 & 11 \\
\hline & & $1^{\mathrm{st} / 2^{\text {nd }}}$ & 0 & 0 & 0 & 0 & 0 & 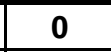 & 1 & 1 & $1^{\text {st } / \text { Last }}$ & 0 & 0 & 2 & 2 & 2 & 0 & 0 & 6 \\
\hline & 12 & $2^{\text {nd }} / 3^{\text {rd }}$ & 0 & 0 & 2 & 2 & 2 & 0 & 1 & 7 & $2^{\text {nd/Last }}$ & 0 & 0 & 2 & 2 & 2 & & 1 & 7 \\
\hline \& & & $3^{\text {rd }} / 4^{\text {th }}$ & 0 & 0 & 0 & 0 & 0 & 0 & 0 & 0 & $3^{\text {rd } / \text { Last }}$ & 0 & 0 & 0 & 0 & 0 & 0 & 0 & 0 \\
\hline & & $1^{\text {st } / 2^{\text {nd }}}$ & 0 & 1 & 0 & 0 & 0 & 1 & 0 & 2 & $1^{\text {st }} /$ Last & 0 & 1 & 0 & 0 & 0 & 1 & 0 & 2 \\
\hline & 13 & $2^{\text {nd }} / 3^{\text {rd }}$ & 0 & 0 & 0 & 0 & 0 & 0 & 0 & 0 & $2^{\text {nd/Last }}$ & 0 & 0 & 0 & 0 & 0 & & 0 & 0 \\
\hline 穴 & & $3^{\text {rd }} / 4^{\text {th }}$ & 0 & 0 & 0 & 0 & 0 & 0 & 0 & 0 & $3^{\text {rd } / \text { Last }}$ & 0 & 0 & 0 & 0 & 0 & 0 & 0 & 0 \\
\hline & & $1^{\mathrm{st} / 2^{\text {nd }}}$ & 0 & 1 & 0 & 0 & 2 & 0 & 0 & 3 & $1^{\text {st/Last }}$ & 0 & 0 & 0 & 0 & 2 & 1 & 0 & 3 \\
\hline & 14 & $2^{\text {nd }} / 3^{\text {rd }}$ & 0 & \begin{tabular}{l|l}
0 \\
\end{tabular} & 0 & 0 & 0 & 0 & 0 & 0 & $2^{\text {nd } / \text { Last }}$ & 0 & 1 & 0 & 0 & 0 & 1 & 0 & 2 \\
\hline & & $3^{\text {rd }} / 4^{\text {th }}$ & 0 & \begin{tabular}{|l|l|}
0 \\
\end{tabular} & 0 & 0 & 0 & 1 & 0 & 1 & $3^{\text {rd } / \text { Last }}$ & 0 & 0 & 0 & 0 & 0 & 1 & 0 & \\
\hline
\end{tabular}


M.S. Amira, et al.,

Table (4): Some physical and chemical properties of studied soil profiles.

\begin{tabular}{|c|c|c|c|c|c|c|c|c|c|c|c|c|c|c|c|}
\hline \multirow{2}{*}{ 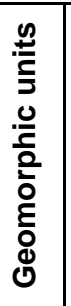 } & \multirow[b]{2}{*}{$\begin{array}{l}z \\
0 \\
\frac{0}{c} \\
\frac{0}{\alpha}\end{array}$} & \multirow[b]{2}{*}{$\begin{array}{l}\text { Depth } \\
\text { cm }\end{array}$} & \multirow[b]{2}{*}{ 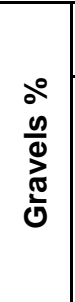 } & \multicolumn{3}{|c|}{$\begin{array}{c}\text { Particle size } \\
\text { distribution \% }\end{array}$} & \multirow[b]{2}{*}{ 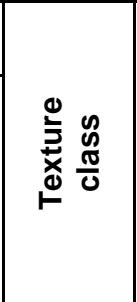 } & \multirow[b]{2}{*}{ 이 } & \multirow[b]{2}{*}{ 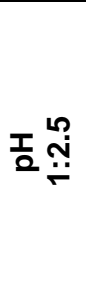 } & \multirow[b]{2}{*}{ 岀 } & \multirow{2}{*}{ 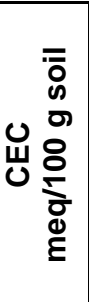 } & \multirow[b]{2}{*}{ ESP } & \multirow[b]{2}{*}{ 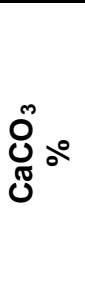 } & \multirow[b]{2}{*}{$\begin{array}{l}\xi \\
\sum_{0}^{5}\end{array}$} & \multirow[b]{2}{*}{$\begin{array}{c}\text { OM } \\
\%\end{array}$} \\
\hline & & & & 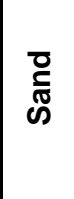 & 节 & $\frac{\widehat{\pi}}{0}$ & & & & & & & & & \\
\hline \multirow{26}{*}{ 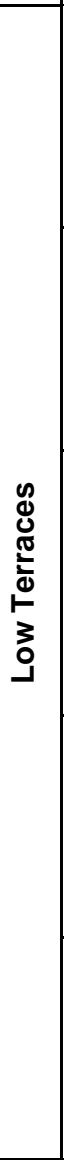 } & & & 5.0 & 84.9 & 5.0 & 10.1 & L sand & 42.4 & 7.4 & 1.8 & 4.9 & 1.2 & 7.2 & 0.67 & 0.60 \\
\hline & & & 4.3 & 82.3 & 5.1 & 12.6 & $L$ sand & 113.1 & 7.3 & 1.0 & 3.9 & 0.6 & 4.9 & 0.17 & 0.40 \\
\hline & 2 & $60-90$ & 3.3 & 84.9 & 2.5 & 12.6 & L sand & $\mid 69.1$ & 7.5 & 0.3 & 4.2 & 0.3 & 0.0 & 0.03 & 0.34 \\
\hline & & $90-120$ & 1.9 & 82.3 & 5.1 & 12.6 & L sand & 122.6 & 7.6 & 0.1 & 3.9 & 0.8 & 0.0 & 0.04 & 0.30 \\
\hline & & W.P.M & 3.6 & 83.6 & 4.4 & 12.0 & L sand & 86.8 & 7.5 & 0.8 & 4.2 & 0.7 & 3.0 & 0.22 & 0.41 \\
\hline & & $0-30$ & 2.5 & 84.9 & 5,0 & 10.1 & L sand & 207.4 & 7.3 & 1.4 & 4.8 & 3.3 & 8.1 & 0.04 & 0.71 \\
\hline & & $30-$ & 3.7 & 84.9 & 5.0 & 10.1 & L sand & 188.6 & 7.2 & 1.7 & 4.2 & 3.7 & 5.0 & 0.03 & 0.60 \\
\hline & 4 & $60-90$ & 2.5 & 84.9 & 2.5 & 12.6 & L sand & 122.6 & 7.6 & 2.6 & 5.7 & 3.4 & 4.1 & 0.01 & 0.55 \\
\hline & & $90-12$ & 2.6 & 82.3 & 2.6 & 15.1 & Sandy L & \begin{tabular}{|l|}
81.7 \\
\end{tabular} & 7.9 & 1.6 & 5.4 & 3.3 & 0.3 & 0.05 & 0.31 \\
\hline & & W.P.M & 2.8 & 84.2 & 3.8 & 12.0 & L sand & 150.1 & 7.5 & 1.8 & 5.0 & 3.4 & 4.4 & 0.03 & 0.54 \\
\hline & & $0-20$ & 13.1 & 79.8 & 5.1 & 15.1 & Sandy L & 14.8 & 7.7 & 0.5 & 5.5 & 0.8 & 9.0 & 0.04 & 0.42 \\
\hline & & $20-$ & 22.1 & 82.3 & 5.1 & 12.6 & sand & 20.4 & 7.6 & 0.2 & 5.0 & 0.6 & 12.6 & 0.03 & 0.20 \\
\hline & 5 & $50-$ & 1.9 & 84.9 & 5.0 & 10.1 & L sand & 103.7 & 7.6 & 0.2 & 5.6 & 0.3 & 3.6 & 0.01 & 0.10 \\
\hline & כ & 80-1 & 1.4 & 84.9 & 5.0 & 10.1 & L sand & 188.6 & 7.5 & 0.1 & 5.1 & 0.3 & 0.8 & 0.01 & 0.33 \\
\hline & & $110-140$ & 1.3 & 79.7 & 5.1 & 15.2 & $L$ sand & 188.6 & 7.5 & 0.3 & 4.2 & 0.2 & 0.9 & 0.03 & 0.20 \\
\hline & & W.P.M & 7.6 & 83.0 & 5.0 & 12.0 & $L$ sand & 109.5 & 7.5 & 0.2 & 5.1 & 0.4 & 5.1 & 0.02 & 0.23 \\
\hline & & $0-3$ & 5.1 & 82.0 & \begin{tabular}{|l|}
7.7 \\
\end{tabular} & 10.3 & sand & 45.3 & 7.4 & 1.2 & 6 & 2.5 & 4.2 & 0.42 & 0.41 \\
\hline & & $30-60$ & 15.0 & 79.1 & 10.4 & 10.5 & sand & 45.3 & 7.2 & 2.0 & 4.5 & 4.0 & 9.3 & .38 & 0.40 \\
\hline & 7 & $0-90$ & 3.6 & 84.5 & 5.2 & 10.3 & sand & 56.6 & 7.3 & 1.8 & 0 & 3.7 & .1 & .09 & 0.40 \\
\hline & & & 6.5 & 86.8 & 2.7 & 10.5 & L sand & 113.1 & 7.3 & 1.0 & 4.2 & 2.5 & 6.0 & 0.10 & 0.20 \\
\hline & & W.P.M & 7.5 & 83.1 & 6.5 & 10.4 & L sand & 65.1 & 7.3 & 1.5 & 3.8 & 3.2 & 6.2 & 0.25 & 0.35 \\
\hline & & $0-2 C$ & 40.6 & 79.6 & 5.1 & 15.3 & Sandy L & 11.5 & 7.9 & 0.5 & 5.6 & 1.2 & 21.5 & 0.03 & 0.41 \\
\hline & & & 27.7 & 81.9 & 5.2 & 12.9 & L sand & 122.6 & 7.8 & 0.8 & 5.4 & 1.1 & 15.3 & 0.04 & 0.30 \\
\hline & 8 & $50-10$ & 15.4 & 84.5 & 5.2 & 10.3 & L sand & |69.1 & 8.0 & 0.1 & 5.2 & 0.6 & 1.4 & 0.03 & 0.40 \\
\hline & & $0-130$ & 3.3 & 86.8 & 2.7 & 10.5 & L sand & 103.7 & 7.5 & 0.1 & 5.0 & 0.5 & 1.6 & 0.02 & 0.30 \\
\hline & & & 19.1 & 83.7 & 4.6 & 11.7 & L sand & 80.6 & 7.8 & 0.3 & 5.3 & 0.8 & 7.7 & 0.03 & 0.35 \\
\hline \multirow{15}{*}{ 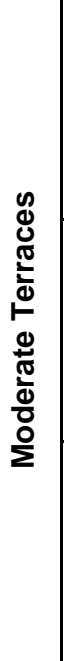 } & & & 14.0 & 84.9 & 5.0 & 10.1 & L sand & 23.6 & 7.9 & 0.3 & 0.6 & 3.0 & 8.2 & .07 & 0.30 \\
\hline & & & & 87.4 & 2.5 & 10.1 & & 122.6 & 7.8 & & & & .6 & 07 & 0.20 \\
\hline & 1 & D-9 & 7.9 & 84.9 & 2.6 & 12.5 & L sand & 245.1 & 7.5 & 0.3 & 6 & 2.7 & 22.2 & 0.01 & 0.21 \\
\hline & & 90 & 3.4 & 84.9 & 5.0 & 10.1 & L sand & 245.1 & 7.4 & 0.1 & 3 & 7 & 6.0 & 0.02 & 0.10 \\
\hline & & W.P.M & 8.4 & 85.5 & 3.8 & 10.7 & $L$ sand & 159.1 & 7.6 & 0.2 & 0.4 & 3.3 & 13.7 & 0.04 & 0.20 \\
\hline & & & 13.0 & 84.9 & 5.0 & 10.1 & L sand & 61.3 & 7.4 & 0.6 & 7 & 0.6 & 6.7 & .13 & 1.30 \\
\hline & & & 12.7 & 82.2 & 2.6 & 15.2 & Sandy L & 51.9 & 7.2 & 1.7 & 4.8 & 1.0 & 13.1 & 1.06 & 0.88 \\
\hline & 3 & & 10.3 & 82.4 & 5.1 & 12.5 & L sand & 226.3 & 7.0 & 0.7 & 3. & 0.3 & 0.1 & 0.06 & 0.10 \\
\hline & & & 12.3 & 84.9 & 5.0 & 10.1 & L sand & 414.9 & 7.3 & 0.8 & & 0.3 & 0.2 & .09 & 0.10 \\
\hline & & W.P.M & 12.1 & 83.6 & 4.4 & 12.0 & L sand & 188.5 & 7.2 & 0.9 & 4.1 & 0.6 & 5.0 & 0.33 & 0.60 \\
\hline & & $0-30$ & 15.9 & 81.8 & 5.2 & 13.0 & L sand & 103.7 & 7.6 & 1.9 & 3.0 & 3.3 & 10.7 & 0.21 & 1.10 \\
\hline & & & 15.5 & 82.0 & 7.7 & 10.3 & L sand & 51.9 & 7.5 & 1.1 & 4.2 & 0.6 & 10.5 & 0.24 & 0.50 \\
\hline & 6 & $60-90$ & 14.8 & 84.5 & 5.2 & 10.3 & L sand & 169.7 & 7.5 & 1.1 & 4.2 & 0.8 & 6.0 & 0.24 & 0.40 \\
\hline & & & 10.7 & 84.6 & 5.2 & 10.2 & L sand & $\mid 69.1$ & 7.6 & 1.2 & 3.6 & 0.7 & 13.1 & 0.16 & 0.20 \\
\hline & & W.P.M & 14.2 & 83.2 & 5.8 & 11.0 & L sand & 98.6 & 7.6 & 1.3 & 3.8 & 1.4 & 10.1 & 0.21 & 0.55 \\
\hline
\end{tabular}


Table (4): Cont.

\begin{tabular}{|c|c|c|c|c|c|c|c|c|c|c|c|c|c|c|c|}
\hline \multirow{2}{*}{ 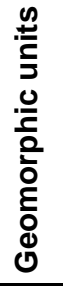 } & \multirow{2}{*}{$\begin{array}{l}\sum_{z} \\
\frac{0}{1} \\
\frac{0}{0} \\
\frac{0}{\alpha}\end{array}$} & \multirow{2}{*}{$\begin{array}{l}\text { Depth } \\
\text { cm }\end{array}$} & \multirow{2}{*}{$\begin{array}{l}\frac{0}{0} \\
\frac{0}{0} \\
\frac{\pi}{0} \\
0\end{array}$} & \multicolumn{3}{|c|}{$\begin{array}{l}\text { Particle size } \\
\text { distribution \% }\end{array}$} & \multirow{2}{*}{ 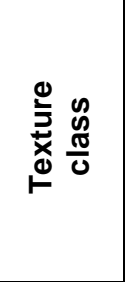 } & \multirow{2}{*}{ 보 } & \multirow{2}{*}{ 工 } & \multirow{2}{*}{ 롱 } & \multirow{2}{*}{ 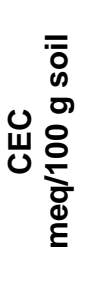 } & \multirow{2}{*}{ ESP } & \multirow{2}{*}{ 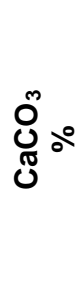 } & \multirow{2}{*}{ 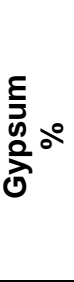 } & \multirow{2}{*}{$\begin{array}{c}\text { OM } \\
\%\end{array}$} \\
\hline & & & & 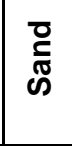 & 竔 & $\frac{\overparen{\pi}}{U}$ & & & & & & & & & \\
\hline \multirow{15}{*}{ 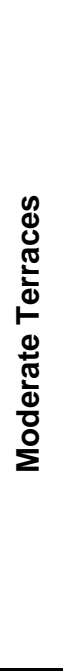 } & \multirow{5}{*}{9} & & 37.9 & 79.2 & 7.8 & 13.0 & L sand & 5.7 & 7.2 & 3.5 & 3.2 & 6.3 & 22.0 & 0.41 & 0.40 \\
\hline & & $30-60$ & 32.8 & 82.0 & 10.3 & 7.7 & L sand & 70.0 & 7.6 & 4.0 & 1.8 & 1.3 & 37.0 & 0.32 & 0.30 \\
\hline & & $60-90$ & 18.0 & 81.5 & 7.9 & 10.6 & L sand & 35.4 & 7.1 & 2.3 & 4.2 & 3.3 & 25.8 & 0.07 & 0.10 \\
\hline & & $90-120$ & 7.6 & 84.0 & 5.3 & 10.7 & L sand & 245.1 & 7.1 & 1.8 & 4.8 & 2.7 & 23.5 & 0.02 & 0.10 \\
\hline & & W.P.M & 24.1 & 81.7 & 7.8 & 10.5 & L sand & 89.1 & 7.2 & 2.9 & 3.5 & 3.4 & 27.1 & 0.20 & 0.13 \\
\hline & \multirow{5}{*}{10} & $0-20$ & 21.4 & |79.1 & 10.4 & 10.5 & L sand & 4.7 & 7.7 & 0.4 & 5.6 & 0.6 & 13.5 & 0.08 & 0.40 \\
\hline & & $20-50$ & 20.1 & 78.5 & 10.7 & 10.8 & L sand & 6.9 & 7.7 & 0.2 & 5.4 & 0.5 & 17.7 & 0.04 & 0.30 \\
\hline & & $50-80$ & 22.4 & 84.1 & 5.3 & 10.6 & L sand & 49.5 & 7.4 & 0.2 & 5.1 & 0.3 & 8.2 & 0.06 & 0.15 \\
\hline & & $80-120$ & 11.2 & 86.3 & 5.5 & 8.2 & Sandy & 163.4 & 7.9 & 0.1 & 5.1 & 0.3 & 0.6 & 0.02 & 0.10 \\
\hline & & W.P.M & 17.9 & 82.6 & 7.6 & 9.8 & L sand & 69.4 & 7.7 & 0.2 & 5.2 & 0.4 & 8.9 & 0.04 & 0.21 \\
\hline & \multirow{5}{*}{11} & $0-30$ & 23.9 & 87.4 & 2.5 & 10.1 & L sand & 25.1 & 7.4 & 2.6 & 4.2 & 4.8 & 12.3 & 0.10 & 0.30 \\
\hline & & $30-60$ & 15.5 & 87.4 & 5.0 & 7.6 & Sandy & 20.7 & 7.3 & 0.4 & 4.2 & 0.3 & 11.3 & 0.08 & 0.30 \\
\hline & & $60-90$ & 12.9 & 84.3 & 5.2 & 10.5 & L sand & 61.3 & 7.3 & 0.2 & 1.5 & 0.7 & 13.3 & 0.09 & 0.21 \\
\hline & & $90-120$ & 4.4 & 84.9 & 5.1 & 10.0 & L sand & 264.0 & 7.5 & 0.6 & 1.8 & 0.2 & 4.1 & 0.07 & 0.20 \\
\hline & & W.P.M & 14.2 & 85.9 & 4.5 & 9.6 & L sand & 92.8 & 7.4 & 0.9 & 2.9 & 1.5 & 10.8 & 0.08 & 0.18 \\
\hline \multirow{15}{*}{ 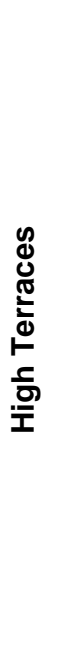 } & \multirow{5}{*}{12} & $0-30$ & $25 . .5$ & 84.9 & 5.0 & 10.1 & L sand & 56.6 & 7.8 & 0.3 & 5.4 & 0.7 & 16.9 & 0.01 & 0.60 \\
\hline & & $30-60$ & 23.4 & 84.9 & 5.0 & 10.1 & L sand & 21.2 & 7.7 & 0.2 & 5.1 & 2.4 & 19.2 & 0.01 & 0.50 \\
\hline & & $60-90$ & 27.0 & 87.4 & 2.5 & 10.1 & L sand & 26.9 & 7.8 & 0.2 & 4.3 & 0.3 & 17.1 & 0.01 & 0.30 \\
\hline & & $90-120$ & 38.1 & 81.9 & 5.2 & 12.9 & L sand & 25.9 & 7.7 & 0.2 & 4.4 & 0.5 & 21.9 & 0.04 & 0.10 \\
\hline & & W.P.M & 28.5 & 84.8 & 4.4 & 10.8 & L sand & 32.7 & 7.7 & 0.2 & 4.8 & 1.0 & 18.7 & 0.02 & 0.23 \\
\hline & \multirow{5}{*}{13} & $0-30$ & 37.6 & 79.6 & 7.6 & 12.8 & L sand & 7.4 & 7.6 & 1.1 & 1.2 & 2.7 & 30.7 & 0.07 & 0.41 \\
\hline & & $30-60$ & 24.5 & 84.7 & 5.1 & 10.2 & L sand & 122.6 & 7.4 & 1.9 & 2.4 & 0.7 & 15.6 & 0.71 & 0.40 \\
\hline & & $60-90$ & 12.9 & 84.8 & 5.0 & 10.2 & L sand & 47.1 & 7.4 & 2.2 & 2.2 & 1.3 & 17.1 & 0.63 & 0.30 \\
\hline & & $90-120$ & 6.0 & 82.4 & 5.0 & 12.6 & L sand & 163.4 & 7.4 & 2.2 & 2.1 & 0.2 & 5.9 & 0.42 & 0.10 \\
\hline & & W.P.M & 20.2 & 82.9 & 5.7 & 11.4 & L sand & 85.1 & 7.5 & 1.9 & 2.0 & 1.2 & 17.3 & 0.46 & 0.20 \\
\hline & \multirow{5}{*}{14} & $0-30$ & 21.9 & 84.6 & 2.6 & 12.8 & L sand & 34.6 & 7.7 & 2.6 & 5.8 & 3.4 & 27.0 & 0.47 & 0.34 \\
\hline & & $30-60$ & 26.6 & 84.9 & 5.0 & 10.1 & L sand & 62.9 & 7.5 & 2.7 & 5.6 & 3.8 & 27.1 & 0.42 & 0.20 \\
\hline & & $60-90$ & 10.0 & 82.4 & 5.0 & 12.6 & L sand & 245.1 & 7.8 & 2.0 & 4.2 & 3.4 & 20.9 & 0.03 & 0.10 \\
\hline & & $90-120$ & 8.2 & 87.2 & 2.6 & 10.2 & L sand & 264.0 & 7.9 & 1.1 & 3.9 & 2.6 & 10.3 & 0.03 & 0.10 \\
\hline & & W.P.M & 15.5 & 85.0 & 3.8 & 11.2 & L sand & 170.8 & 7.7 & 2.0 & 4.7 & 3.2 & 19.9 & 0.20 & 0.17 \\
\hline
\end{tabular}

\section{Soil classification}

The studied soils were classified on the basis of morphological descriptions, physical and chemical properties with respect to the meteorological data of the studied area. The dominant soil moisture regime in this area is Torric with Thermic soil temperature regime. All the soils haven't any diagnostic horizon within $1 \mathrm{~m}$ from the surface. Therefore, they could be affiliated to Entisols order according to Soil Survey Staff (2014). Moreover, they classified up to sub great group level as Typic Torripsamments.

\section{REFERENCES}

Abdel-Gaffar, A. S., M. S. Marei and H. M. Gaber (1997). Integrated land development of southern Egypt: Available resources and alternative 
options. Inter. Dev. Res. Center, Cairo \& Soil and Water Sci. Dept., Alex. Univ.

Ashoub, T.M. (1996). Water use technology in Wadi El-natrun soils. Ph.D. Thesis, Fac. Agric., Cairo Univ.

Bilzi, A. F. and E. J. Ciolkosz (1977). A field morphology rating scale for evaluating pedological development. Soil Sci. 124: 45-48.

Burt, Rebecca and Soil Survey Staff (2014). Kellogg Soil Survey Laboratory Methods Manual, Soil Survey Investigations Report No. 42, Version 5.0, Kellogg Soil Survey Laboratory, National Soil Survey Center, Natural Resources Conservation Service, USDA, Lincoln, Nebraska, USA.

Dehaan, R. L. and G. R. Taylor (2003). Image-derived spectral endmembers as indicators of salinization. International Journal of Remote Sensing, 24(4): 775-794.

Erian, W.F. (1989). Soil climate and balance diagram of some horizontal expansion areas in Egypt. Egypt. J. Soil Sci., Special Issue: $\mathbf{4 5 - 7 4}$

ESRI "Environmental Systems Research Institute" (2003). Using ArcGIS Geostatistical Analyst. Environmental Systems Research Institute (ESRI) Press, Redlands, California.

ESRI "Environmental Systems Research Institute" (2014). Arc Map Version 10.1
User Manual. ESRI, 380 New York Street, Redlands, California, 923738100, USA.

FAO (2006). Guidelines for soil profile description. Soil Res. Dev. and Co. Serv., Land and Water Dev. Div., Rome, Italy.

ITT "International Telephone \& Telegraph" (2012). ITT corporation ENVI 5 software, 1133 Westchester Avenue, White Plains, NY 10604, USA.

Klute, A. and C. Dirksen (1986). Hydraulic conductivity and diffusivity laboratory methods. Part 1. Physical and mineralogical methods. American Society of Agronomy, Madison pp. 687-734.

Lillesand, T. M. and R. W. Kiefer (2007). Remote Sensing and Image Interpretation. 5th Ed. Paper back. John Wiley, New York.

Munsell Color (1992). Munsell Color Charts, Munsell Color Company, Baltimore, Maryland.

Quinn, S. (1998). Egypt's Toshka project presented to U.S., Arab World Online., http://www.awo.net/tradelin. October 2, 1998.

Soil Survey Staff (2014). Keys to Soil Taxonomy, $11^{\text {th }}$ Ed., USDA, NRCS, Pocahontas Press, Inc., Blacksburg, Virginia, USA. 
تكامل المعلومات الجغرافية والاستشعار عن بعد في دراسة الخصائص الجيومورفولوجية

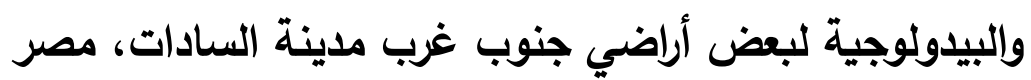

محمد سمير عراقي عميرة(")، محمد حسن بهنسي(")، وائل محمد ندا(")،

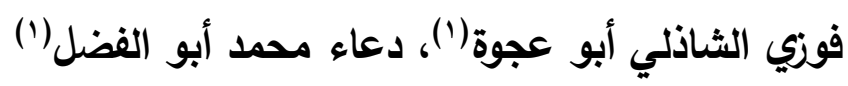

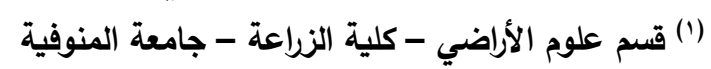

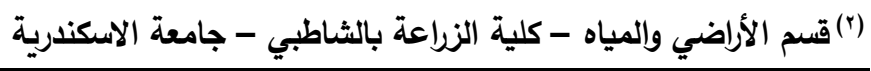

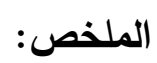

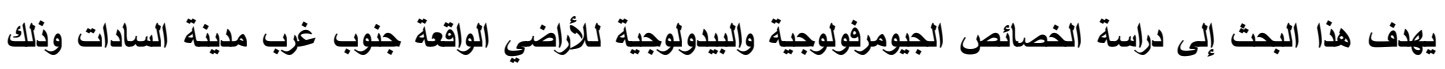

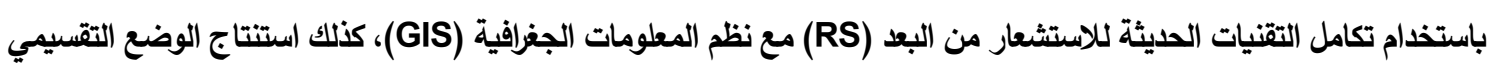

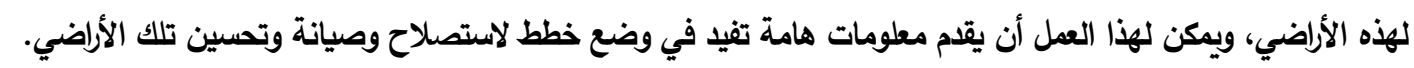

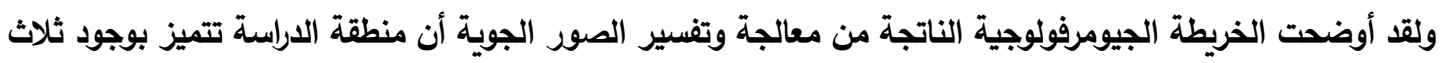

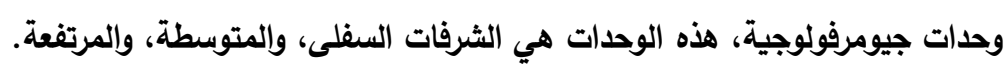

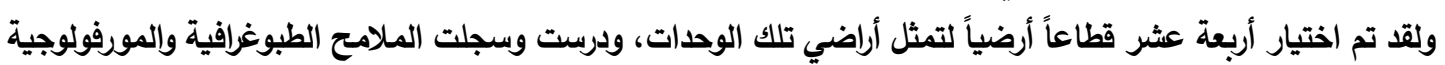

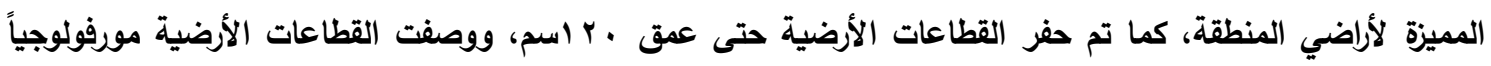
وأخذت منها عينات ممثلة للأفاق المختلفة لإجراء التحليلات المعملية لتقدير الخواص الطناع الطبيعية والكيماوية.

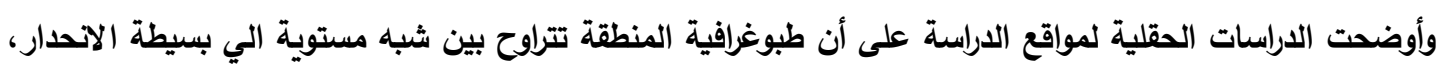

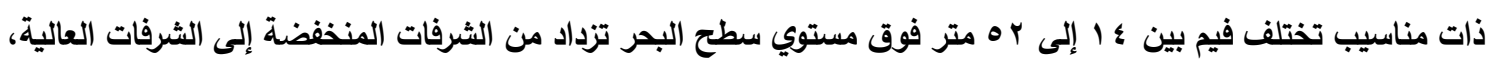
والأراضي عميقة، وحالة الصرف جيدة. قوام هذه الأراضي يغلب عليه الطمي رملي تتزيد فيه نسبة الحصى من الثرفات المنففضة إلى العليا، والتوصيل

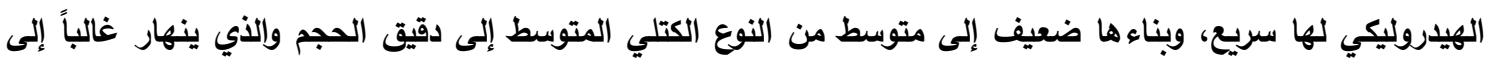
الحبيبي مع وجود طبقات رملية مفككة.

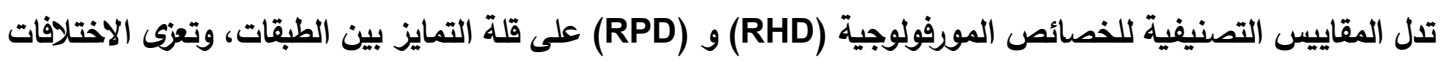

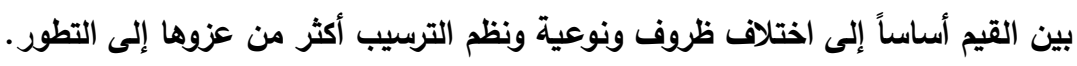

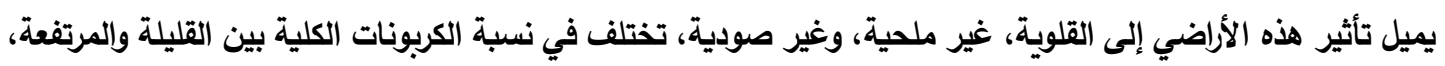

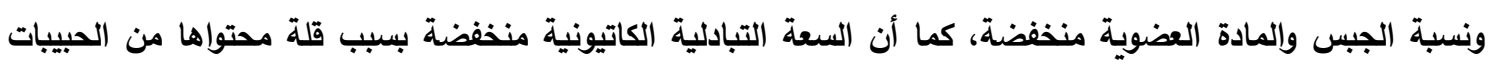
الاقيقة والمادة العضوية. ونظراً لأنه لم يتضح في هذه الأراضي أي آفاق تثخيصية فلقد تم تقسيم تلك الأراضي تبعا للتقسيم الأمريكي الحديث

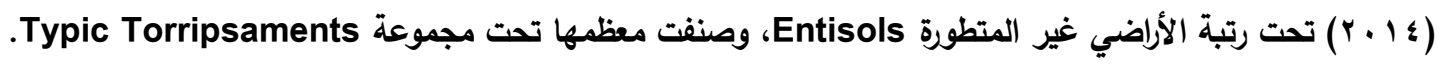

الكلمات الدالة:

الاستشعار من البعد، نظم المعلومات الجغرافية، الوحدات الجيومورفولوجية، تقسيم الأراضي.

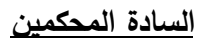

أ.د/ أحمد عبدالفتاح البــــارودى كلية الززاعة - جامعة طنطا

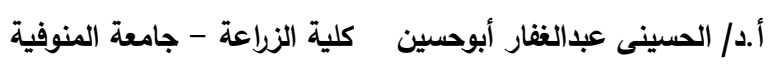

